\title{
(In)segurança alimentar no Brasil no pré e pós pandemia da COVID-19: reflexões e perspectivas ${ }^{1}$
}

\author{
Bruna Fernanda do Nascimento Jacinto de Souza² \\ Milena Serenini Bernardes ${ }^{3}$ \\ Valéria Cristina Ribeiro Vieira ${ }^{4}$ \\ Priscila Maria Stolses Bergamo Francisco ${ }^{1}$ \\ Letícia Marín-León ${ }^{1}$ \\ Daniele Flaviane Mendes Camargo' \\ Ana Maria Segall Corrêa ${ }^{1}$
}

\section{RESUMO}

A alimentação e a nutrição adequadas são fundamentais para a promoção e a proteção da saúde, podendo determinar as condições de saúde de indivíduos e coletividades de diversos modos. A despeito dos avanços conquistados no Brasil - no que tange à realização do direito humano à alimentação adequada (DHAA) e à concretização da segurança alimentar e nutricional (SAN) - vêm-se observando, desde 2016, o enfraquecimento e o desmonte das políticas públicas de garantia de direitos e fortalecimento de sistemas alimentares sustentáveis. Tal cenário contribui para o aumento da pobreza e a piora das condições de vida de consideráveis parcelas da população. Os dados da última Pesquisa de Orçamentos Familiares (POF 2017-2018) mostram que a prevalência de insegurança alimentar no país aumentou para 36,1\% (era 22,9\% em 2013), sendo que 3,1 milhões de famílias vivenciaram a experiência da fome no período analisado. Essa situação se agrava em função da crise política e da crise sanitária causada pela pandemia da COVID-19. Nesse contexto, torna-se premente a reflexão sobre quais são as perspectivas no país em relação à garantia do DHAA e da SAN, especialmente para os grupos mais vulneráveis, considerando dimensões como acesso à alimentação, renda básica, programas de transferência de renda, sistemas e ambientes alimentares e mecanismos de participação e controle social.

Termos de indexação: Segurança Alimentar e Nutricional; Programas e Políticas de Nutrição e Alimentação; Política Pública; Coronavírus; Pandemia; COVID-19.

\footnotetext{
${ }^{1}$ Artigo baseado na tese de BFNJ SOUZA, intitulada "Insegurança alimentar em domicílios de Campinas/SP: características demográficas, socioeconômicas e de alimentação". Universidade Estadual de Campinas; 2020. Tese de doutorado defendida em 03/07/2020.

${ }^{2}$ Departamento de Saúde Coletiva. Faculdade de Ciências Médicas. Universidade Estadual de Campinas. Rua Tessália Vieira de Camargo, 126 Cidade Universitária Zeferino Vaz. Barão Geraldo. Campinas, SP, Brasil. Correspondência para: BFNJ SOUZA. E-mail: brunafnjs@hotmail.com

${ }^{3}$ Universidade Federal de São Paulo, Departamento de Pediatria, São Paulo, SP, Brasil

${ }^{4}$ Universidade Federal de Alfenas, Faculdade de Nutrição, Alfenas, MG, Brasil
} 


\section{INTRODUÇÃO}

A alimentação e a nutrição adequadas são fundamentais para a promoção e a proteção da saúde, podendo determinar as condições de saúde de indivíduos e coletividades de diversos modos [1]. No Brasil, o direito humano à alimentação adequada (DHAA) tem sido objeto de debate pelas sociedades acadêmicas e civis desde a década de 80, englobando dois importantes aspectos legais: os direitos à alimentação adequada e de estar livre da má nutrição e da fome [2,3].

Os principais inquéritos de alimentação e nutrição realizados no país desde a década de 1970 demonstraram a transição nutricional em processo e sua relação com a modificação nos padrões de consumo alimentar, associada às mudanças nos sistemas alimentares. Os inquéritos mostraram progressiva redução da desnutrição e aumento nas prevalências de excesso de peso e obesidade, com consequente crescimento das doenças crônicas não transmissíveis (DCNT) $[4,5]$. As mudanças na alimentação dos brasileiros apontaram diminuição do consumo de alimentos tradicionais - como arroz, feijão, hortaliças, leguminosas, raízes e tubérculos - e aumento do consumo de alimentos ultraprocessados (AUP) como biscoitos e refrigerantes [5-7].

Mais recentemente, instituições brasileiras vêm buscando responder às diferentes formas de má nutrição de maneira integrada. Apesar disso, o país continua marcado por iniquidades em saúde e alimentação, cujos impactos negativos são mais sentidos em grupos como crianças, adolescentes, mulheres, beneficiários de programas de transferência de renda (PTR), povos indígenas, quilombolas e, sobretudo, entre os mais pobres e de raça/cor negra [8-18].

A partir de 2003, o governo brasileiro ampliou e instituiu políticas e programas de combate à fome e promoção do DHAA. Investimentos foram direcionados ao desenvolvimento de programas intersetoriais com interfaces entre produção agropecuária, combate à pobreza, acesso à alimentação nas escolas, distribuição de alimentos em comunidades vulneráveis e educação alimentar e nutricional [19].

Em 2006, foi sancionada a Lei Orgânica de Segurança Alimentar e Nutricional (LOSAN), que estabeleceu princípios, diretrizes e objetivos do Sistema Nacional de Segurança Alimentar e Nutricional (SISAN). Nela é reforçada a alimentação adequada como direito fundamental e o dever do poder público de promover e prover o DHAA, assim como garantir mecanismos para sua exigibilidade [20-21]. A LOSAN definiu o conceito de Segurança Alimentar e Nutricional (SAN) como o direito de todo cidadão "ao acesso regular e permanente a alimentos de qualidade, em quantidade suficiente, sem comprometer o acesso a outras necessidades essenciais, tendo como base práticas alimentares promotoras de saúde que respeitem a diversidade cultural e que sejam ambiental, cultural, econômica e socialmente sustentáveis" [21]. Em contraposição, a insegurança alimentar (IA) "existe quando as pessoas não têm acesso econômico, social e físico adequado a quantidades suficientes de alimentos seguros e nutritivos para o crescimento e desenvolvimento normais e uma vida ativa e saudável" [22].

Nesse sentido, é possível reconhecer a desnutrição e a obesidade como expressões distintas da insegurança alimentar e nutricional [23]. Existem evidências de coexistência de IA e obesidade em um mesmo território e até em um mesmo domicílio, associada à falta de dinheiro ou outros meios para obtenção de alimentos.

Nessas circunstâncias, indivíduos e famílias desenvolvem estratégias de enfrentamento da carência alimentar e da fome, incluindo adoção de dietas baseadas em alimentos de baixos custo e qualidade e alta densidade energética, como os AUP [24-26].

Para monitorar o impacto das políticas de SAN implementadas no Brasil, validou-se em 2003 uma escala psicométrica de medida direta e domiciliar da segurança e insegurança alimentar, a Escala Brasileira de Insegurança Alimentar (EBIA), instrumento que identifica desde o componente psicológico da IA, traduzido pela preocupação de que a comida possa vir a faltar, até sua situação extrema - que é a experiência da fome [27]. Em 2004, realizou-se o primeiro diagnóstico com abrangência e representatividade nacionais, por meio da incorporação da EBIA ao Suplemento de Segurança Alimentar da Pesquisa Nacional por Amostra de Domicílios (PNAD2004) [28].

Foram analisadas as tendências temporais e fatores associados à IA no Brasil a partir dos microdados de três PNAD's consecutivas (2004, 2009 e 2013). Observouse aumento na prevalência de segurança alimentar (SA) de 64,8\% em 2004 para 77,1\% em 2013 e redução na prevalência de IA, especialmente da IA moderada e da grave que, juntas, passaram de $17 \%$ em 2004 para 7,9\% em 2013 [29].

Em 2014, o Brasil atingiu a meta dos Objetivos de Desenvolvimento do Milênio de reduzir pela metade a 
prevalência de subnutrição e ineditamente ficou fora do "Mapa da Fome" [30]. A Organização das Nações Unidas para Alimentação e Agricultura (FAO) destacou o crescimento econômico inclusivo, com melhores oportunidades para os pobres, aprimoramento da produtividade dos agricultores familiares e reforço da proteção social como fatores relacionados ao sucesso nessa redução [31]. No Brasil, também foram destacados fortalecimento do poder aquisitivo das mulheres, PTR e melhoria da renda dos mais pobres [32].

A despeito desses avanços, desde 2016 e com especial aprofundamento a partir de 2018, vêm-se observando o enfraquecimento e o desmonte das políticas públicas de garantia de direitos e fortalecimento de sistemas alimentares sustentáveis. Tal cenário contribui para o aumento da pobreza e a piora das condições de vida de consideráveis parcelas da população $[23,33]$. Resultados recentes da Pesquisa de Orçamentos Familiares (POF 2017-2018) mostraram que a prevalência de IA no país aumentou para $36,1 \%$ e que 3,1 milhões de famílias vivenciaram, naquele período, a experiência da fome [34].

Em março de 2020, a Organização Mundial da Saúde declarou como pandemia o que era, inicialmente, um surto da doença causada pelo novo coronavírus (COVID-19) restrito à cidade de Wuhan na China, que rapidamente se espalhou para os outros continentes [35]. Na América Latina, o Brasil é o país com maiores registros de infecções e óbitos [36].

À essa crise mundial da saúde soma-se a crise econômica, ocasionando aumento de desemprego, desigualdade social e do risco de crise alimentar. Relatório do Comitê Mundial de Segurança Alimentar apontou que a disponibilidade de alimentos está sendo afetada em curto e longo prazos e que os mais pobres e demais atingidos pela recessão poderão ter o acesso aos alimentos e qualidade da alimentação comprometidos [37]. Resultados preliminares do Estudo Nutrinet Brasil mostraram que, em geral, houve aumento no consumo de hortaliças, frutas e feijão no período da pandemia, mas também aumentou o consumo de AUP nas regiões Norte e Nordeste, e entre pessoas de menor escolaridade [38]. Pesquisa realizada pela Empresa Brasileira de Pesquisa Agropecuária (EMBRAPA) apontou que, embora as hortaliças continuem acessíveis para compra durante a quarentena, maior consumo é observado entre indivíduos de maior renda e das regiões Sul e Sudeste [39].

Inquérito recente realizado pelo Fundo das Nações Unidas para a Infância (UNICEF) destaca o impacto da pandemia nas famílias brasileiras com crianças e adolescentes (C/A). A proporção dos entrevistados que residem com C/A e relatou que os hábitos alimentares mudaram nas suas casas foi de $58 \%$. Houve maior consumo de AUP como macarrão instantâneo, biscoitos recheados, refrigerantes e alimentos típicos de fastfood. Além disso, 21\% passou por momentos em que os alimentos acabaram e não havia mais dinheiro para reposição, e $6 \%$ deixou de fazer uma refeição por falta de dinheiro, sendo que as proporções foram maiores entre entrevistados que residem com C/A, nas regiões Norte e Nordeste, e nas famílias com menor renda [40].

Nesse sentido, torna-se premente a reflexão sobre quais são as perspectivas no país em relação à garantia do DHAA e da SAN, especialmente para os grupos mais vulneráveis, considerando dimensões como acesso à alimentação, renda básica/PTR; sistemas e ambientes alimentares e mecanismos de participação e controle social. A seguir são apresentadas contextualizações das principais iniciativas de políticas de Estado no Brasil que objetivaram reduzir a IA e garantir o DHAA.

\section{Renda Básica e Programas de Transferência de Renda}

A renda monetária é considerada um preditor da SAN, sendo os PTR, como o Programa Bolsa Família (PBF), fundamentais, portanto, para a redução da IA e da fome $[14,41]$. Assim, juntamente com o crescimento da economia e aumento real do salário mínimo, o progressivo aumento da cobertura dos PTR teve papel relevante para o acesso à alimentação adequada e a melhoria das condições de vida, especialmente entre famílias em situação de extrema pobreza [41]. Estudo realizado entre 2011-2014 em município do Nordeste brasileiro com alta proporção de famílias em pobreza/extrema pobreza observou que ao longo do período acompanhado, 24,5\% daquelas que viviam em condição de IA migraram para SA e que, se o PTR não estivesse em vigor, aproximadamente $10 \%$ dessas famílias teriam permanecido em IA [42]. Não obstante tal relevância, o aumento do desemprego e, portanto, queda da renda média das famílias, em 2019 o governo iniciou corte de gastos com o PBF, reduzindo o número de beneficiários [43].

A pandemia comprometeu diretamente o rendimento de milhões de brasileiros, com impacto maior nos setores econômicos que empregam proporcionalmente mais mulheres, negros, trabalhadores informais, pessoas que ganham menores salários e de famílias mais pobres [43]. 
No inquérito do UNICEF, entre os entrevistados que residem com C/A, $21 \%$ estava trabalhando e ficou desempregado e $63 \%$ referiu diminuição dos rendimentos familiares (em $25 \%$ a renda foi reduzida à metade) [40].

Em março de 2020, o Congresso Nacional aprovou o auxílio Renda Básica Emergencial (RBE) (PL 9236/17) para atender pessoas de baixa renda, com maioridade legal, sem emprego formal e com renda familiar mensal de até três salários mínimos, estipulando-se o valor de $R \$ 600,00$, com o teto de dois benefícios por família [44]. A RBE substitui o PBF nos casos em que for mais vantajoso receber o auxílio emergencial. Simulações realizadas pelo Insper apontam a potencialidade da RBE para redução da pobreza, com impactos maiores em cenários onde é direcionada para o público mais vulnerável [43].

Segundo Burity e cols (2010) [45], por requisito constitucional, o Estado brasileiro deve prover o DHAA, especialmente para indivíduos e grupos com risco maior de $I A$, associando ações de transferência de renda ou renda básica à entrega de alimentos, de acordo com as especificidades de cada grupo ou comunidade, ou outras formas de seguridade social [45].

\section{Programa de Aquisição de Alimentos e modelos de produção}

O Programa de Aquisição de Alimentos (PAA) é um mecanismo de compra direta de alimentos da agricultura familiar. A compra com doação simultânea é sua principal modalidade, a partir da qual os alimentos adquiridos são repassados para equipamentos públicos de SAN e/ou entidades da rede socioassistencial. Criado no âmbito do Programa Fome Zero, o PAA tornou-se importante estratégia de fortalecimento da agricultura familiar e promoção do acesso à alimentação, sobretudo para populações em IA [46]. Aproximadamente $63 \%$ dos alimentos adquiridos pelo PAA são in natura, e outros $22,5 \%$ são minimamente processados (fubá, por exemplo), dados que reforçam seu potencial para a promoção da alimentação adequada e saudável [47]. Segundo a FIAN-Brasil (2019), o PAA promove o fortalecimento de sistemas alimentares locais, estimulando a produção da agricultura familiar, priorizando processos agroecológicos, opostamente ao modelo do agronegócio, voltado para as principais cadeias de commodities alimentares [48].

Desde 2016, o PAA vem sofrendo profundos cortes orçamentários, que se intensificaram nos últimos anos, colocando em risco a existência do programa. Tais reduções podem provocar impactos em outras políticas de promoção da SAN, como no Programa Nacional de Alimentação Escolar (PNAE). O PAA promove a inclusão produtiva dos agricultores mais pobres e simultaneamente garante acesso à alimentação saudável, apresentando-se, portanto, como estratégia fundamental para mitigar os impactos sociais e econômicos da pandemia. Ressalta-se que a pandemia aumentou o risco de agravamento da $I A$, que pode favorecer a redução da imunidade, com aumento do risco de ocorrência e evolução grave do quadro clínico do novo coronavírus [40].

Diante disso, orientações de instituições internacionais e nacionais para o momento pós-pandemia reforçam a importância de políticas públicas que garantam produção doméstica de alimentos e renda ao agricultor, promovendo SAN também no campo [49-51]. Um investimento de R\$1 bilhão no PAA, ainda em 2020, beneficiaria 208 mil produtores familiares, em 2.647 municípios, que poderiam produzir 420 mil toneladas de alimentos [47]. Análise realizada pelo Instituto de Pesquisa Econômica Aplicada (IPEA) mostrou que o PAA promove dinamização das economias locais e que - nos anos em que recebeu maiores investimentos (2011-2012) - observou-se maior crescimento do Produto Interno Bruto nos municípios que acessaram esses recursos [47].

Igualmente imprescindível é a manutenção da destinação mínima de $30 \%$ dos recursos do Fundo Nacional de Desenvolvimento da Educação para aquisição de gêneros alimentícios diretamente da agricultura familiar e sua utilização para compor cestas básicas entregues aos estudantes durante o período de distanciamento físico [52].

Considerando a interrupção das aulas, importante medida contra a disseminação da COVID-19, e tendo em vista a relevância do PNAE para garantia da SAN, o governo brasileiro lançou cartilha de orientação sobre a continuidade do programa durante a pandemia [53]. Municípios que possuíam redes bem estruturadas de SAN se destacaram na proposição de ações em tempo oportuno para manter a alimentação dos alunos das escolas públicas e evitar o agravamento da IA. A entrega de kits com alimentos in natura para as famílias é uma estratégia que assegura qualidade nutricional da alimentação e possibilita manter a compra da agricultura familiar [54-56]. Outro exemplo é o "Cartão Merenda" da Prefeitura de São Paulo que transfere recursos para a compra de alimentos pelas famílias, dependendo da etapa de ensino do aluno [57]. 


\section{Sistemas e ambientes alimentares}

Os sistemas alimentares estão associados à transição nutricional. Eles compreendem todos os processos relacionados à alimentação, desde o cultivo e colheita, passando pelo processamento, distribuição, publicidade e marketing, preparação e consumo de alimentos, bem como o manejo do descarte das sobras $[58,59]$. Os ambientes alimentares compreendem as dimensões política, econômica, sociocultural e física em que os indivíduos se relacionam com o sistema alimentar e que influenciam suas escolhas alimentares e estado nutricional. Eles mediam, portanto, os sistemas e as práticas alimentares, de forma que sua organização pode condicionar deterioração da saúde e aumento das desigualdades sociais. Essa organização inclui as normas políticas, legais e culturais de um território; recursos financeiros disponíveis; e os espaços físicos onde os indivíduos obtém os alimentos, ou seja, a disponibilidade de estabelecimentos de comercialização como hortas, varejões, supermercados, restaurantes, lanchonetes, que tem relação com o preço e a qualidade dos alimentos $[23,60]$.

O sistema alimentar globalizado hegemônico intensificou a produção e a oferta de AUP, fabricados a base de matérias-primas muito baratas. Os AUP estão diretamente relacionados com a degradação ambiental, pela extensiva utilização de monoculturas. Os sistemas alimentares tradicionais, com base na agricultura familiar, estão sendo suprimidos pelo agronegócio, transnacionais e hipermercados que passaram a dominar a produção/ oferta de AUP e ameaçar as culturas alimentares locais, especialmente nos países de baixa renda $[6,38,61]$. Nesses países, o consumo de AUP pode substituir o de alimentos in natura ou minimamente processados. Esse deslocamento tem impacto direto no aumento da prevalência da obesidade e das principais DCNT [61], consideradas fatores de risco para manifestações mais graves da COVID-19 [62].

No período da pandemia, tem-se observado maior consumo de AUP em virtude de seu baixo custo, apresentação e disponibilidade [38]. Embora o conceito de SAN disposto na LOSAN não faça menção literal a esses alimentos, um dos componentes da IA em nível domiciliar e individual é a qualidade da dieta, a qual é prejudicada pelo teor nutricional inadequado dos alimentos.

Conselho Nacional de Segurança Alimentar e Nutricional
Os avanços na agenda de combate à fome e promoção da SAN são frutos da intensa mobilização e engajamento da sociedade civil brasileira, sendo o Conselho Nacional de Segurança Alimentar e Nutricional (CONSEA) protagonista desse processo. Enquanto órgão consultivo, de fiscalização, planejamento e avaliação, o CONSEA apresenta caráter interdisciplinar e transversal, que considera as diversas realidades sociais, congregando diferentes setores na defesa do DHAA. No entanto, em janeiro de 2019, no primeiro dia da nova gestão federal, foi publicada a Medida Provisória № 870 que extinguiu - CONSEA, colocando fim na principal instância de participação da sociedade civil nas políticas públicas de SAN [21].

Apesar de a extinção do CONSEA ter fragilizado drasticamente a agenda de políticas públicas relacionadas à SAN, sua ausência não impediu a articulação da sociedade para a proposição de diretrizes para o enfrentamento da situação emergencial ora enfrentada. A sociedade civil brasileira foi mais uma vez convocada para atuar na defesa da SAN frente à crise sanitária provocada pelo novo coronavírus. Destaca-se, nesse sentido, a atuação da comissão organizadora da I Conferência Nacional, Popular e Autônoma: por Direitos, Democracia e Soberania e Segurança Alimentar e Nutricional, e o Fórum Brasileiro de Soberania e Segurança Alimentar e Nutricional que, juntos a diversas outras organizações, colaboraram com a produção de documentos salientando as prioridades para o enfrentamento da IA nesse contexto. A criação de Comitês Emergenciais de Combate à Fome merece destaque enquanto proposta que viabiliza a possibilidade de participação da sociedade na tomada de decisão sobre as ações relacionadas à SAN [63].

A pandemia acirrou as desigualdades sociais no Brasil, com o agravante da demora na proposição de medidas e diretrizes nacionais para o enfrentamento da IA. Evidenciase, mais uma vez, a importância do CONSEA e de todas as formas de interlocução da sociedade com o Estado, enquanto princípio fundamental para a democracia, amparado constitucionalmente [64]. Especialmente nesse momento, é importante que a sociedade exerça seu direito de controle social e de participação na elaboração e implementação de políticas e ações para garantia da SAN. Diante desse contexto, urge a reativação/criação e o fortalecimento de Conselhos Municipais/Estaduais de SAN, além de outros espaços de participação popular. 


\section{COMENTÁRIOS FINAIS}

O aumento da pobreza e das desigualdades, especialmente relacionadas às mudanças na ocupação e rendimento - num contexto de múltiplas crises - piora as condições de vida da população, com impactos diretos na alimentação.

Os dados da última POF (2017-2018) e outras publicações mostraram piora nas condições de SAN da população brasileira, tanto em função das crises política e econômica iniciadas em 2015 e agravadas a partir daí [34,65,66], quanto em decorrência da crise sanitária da COVID-19. Por essa razão cresce a percepção e o receio de que o Brasil volte a figurar no "Mapa da Fome".

A capacidade de gestão dos recursos financeiros e a natureza e eficácia das políticas públicas existentes nessas áreas, incluindo a previdência social, estão diretamente relacionadas à forma como as localidades podem ser afetadas pelas consequências da crise sanitária. De forma que as decisões políticas podem determinar se as desigualdades serão ou não agravadas, sendo a liderança do mais alto nível do governo crucial para evitar esse retrocesso, e garantir que os direitos sejam priorizados $[38,67]$.

\section{REFERÊNCIAS}

1. Brasil. Lei $n^{\circ}$ 8.080, de 19 de setembro de 1990 . Dispõe sobre as condições para a promoção, proteção e recuᄀperação da saúde, a organização e o funcionamento dos serviços correspondentes e dá outras providências. Diُário Oficial da União, Brasília, 19 set.

2. Leão M. A construção social de um sistema público de segurança alimentar e nutricional: a experiência brasileira. Brasília: ABRANDH; 2012.

3. United Nations Human Rights. Office of the high commissioner for human rights. Food and Agriculture Organization of the United Nations. The right to adequate food. Human rights. Geneva: United Nations Human Rights; 2010

4. Instituto Brasileiro de Geografia e Estatística. Pesquisa de Orçamentos Familiares 2008-2009. Antropometria e estado nutricional de crianças, adolescentes e adultos no Brasil. Rio de Janeiro: IBGE; 2010 [citado 2020 Ago 20]. Disponível em: https://biblioteca.ibge.gov.br/visualizacao/livros/liv45419.pdf
5. Schmidt MI, Duncan BB, Silva GA, Menezes AM, Monteiro CA, Barreto SM, et al. Chronic non-communicable diseases in Brazil: burden and current challenges. Lancet. 2011; 377:194961.

https://doi.org/10.1016/S0140-6736(11)60135-9

6. Souza NP, Lira PIC, Fontbonne A, Pinto FCL, Cesse EAP. A (des)nutrição e o novo padrão epidemiológico em um contexto de desenvolvimento e desigualdades. Cien Saude Colet. 2017; 22(7): 2257-2266. http:// dx.doi.org/10.1590/1413-81232017227.03042017

7. Instituto Brasileiro de Geografia e Estatística. Pesquisa de Orçamentos Familiares 2017-2018: Análise do consumo alimentar pessoal no Brasil. Rio de Janeiro: IBGE; 2020 [citado 2020 Set 10]. Disponível em: https:// biblioteca.ibge.gov.br/visualizacao/livros/liv101742.pdf

8. Fávaro T, Ribas DLB, Zorzatto JR, Segall-Corrêa AM, Panigassi G. Segurança alimentar em famílias indígenas Teréna, Mato Grosso do Sul, Brasil. Cad Saude Publica. 2007; 23(4):785793. http://dx.doi.org/10.1590/50102-311X2007000400006

9. Afonso LFC, Corrêa NAF, Silva HP. Segurança Alimentar e Nutricional em comunidades quilombolas no Brasil: um balanço da literatura indexada. Segur Aliment Nutr. 2019; 27:113, e020003. https://doi.org/10.20396/san.v27i0.8652861

10. Silva EKP, Medeiros DS, Martins PC, Sousa LA, Lima GP, Rêgo MAS, et al. Insegurança alimentar em comunidades rurais no Nordeste brasileiro: faz diferença ser quilombola? Cad Saude Publica. 2017; 33(4):e00005716. https://doi.org/10.1590/0102-311×00005716

11. Souza BFNJ, Marin-Leon L, Camargo DFM, SegallCorrêa AM. Demographic and socioeconomic conditions associated with food insecurity in households in Campinas, SP, Brazil. Rev Nutr. 2016; 29(6):845-57. http://dx.doi.org/10.1590/1678-98652016000600009

12. Guerra LDS, Espinosa MM, Bezerra $A C D$, Guimarães LV, Lima-Lopes MA. Insegurança alimentar em domicílios com adolescentes da Amazônia Legal Brasileira: prevalência e fatores associados. Cad Saude Publica. 2013; 29(2):335348. https://doi.org/10.1590/s0102-311X2013000200020

13. Werneck J. Racismo institucional e saúde da população negra. Saude Soc. 2016; 25(3):535549. $\quad$ https://doi.org/10.1590/s0104-129020162610 
14. Marin-Leon L, Francisco PMSB, Segall-Corrêa AM, Panigassi G. Bens de consumo e insegurança alimentar: diferenças de gênero, cor de pele autorreferida e condição socioeconômica. Rev Bras Epidemiol. 2011; 14(3):398-410. http://dx.doi.org/10.1590/S1415-790X2011000300005

15. Panigassi G, Segall-Corrêa AM, Marin-León L, PérezEscamilla R, Sampaio MFA, Maranha LK. Insegurança alimentar como indicador de iniquidade: análise de inquérito populacional. Cad Saude Publica. 2008;24(10):2376-2384 http://dx.doi.org/10.1590/s0102-311X2008001000018

16. Lignani JB, Sichieri R, Burlandy L, SallesCosta R. Changes in food consumption among the Programa Bolsa Família participant families in Brazil. Public Health Nutr. 2010; 14(5):78592. $\quad$ https://doi.org/10.1017/S136898001000279X

17. Santos LMP, Carneiro FF, Hoefel MGL, Santos W, Nogueira TQ. O meio de vida precário em lixões: um relatório sobre insegurança alimentar e fome entre catadores de materiais recicláveis. Rev Nutr. 2013; 26 (3):323-334. https://doi.org/10.1590/s1415-52732013000300007

18. Instituto Brasileiro de Geografia e Estatística. Desigualdades sociais por cor ou raça no Brasil. Estudos e Pesquisas. Informação demográfica e socioeconômica. n.41. Rio de Janeiro: IBGE; 2019 [citado 2020 Ago 20]. Disponível em: https://biblioteca.ibge. gov.br/visualizacao/livros/liv101681 informativo.pdf

19. Belik W. Segurança Alimentar e Nutricional e o Direito HumanoàAlimentação. RevSociol Polit. 2012;19(2):95-110.

20. Albuquerque MFM. A segurança alimentar e nutricional e o uso da abordagem de direitos humanos no desenho das políticas públicas para combater a fome e a pobreza. Rev Nutr. 2009; 22(6):895-903. http://dx.doi.org/10.1590/s1415-52732009000600011

21. Castro IRR. A extinção do Conselho Nacional de SegurançaAlimentare Nutricionaleaagendadealimentação e nutrição. Cad Saude Publica. 2019; 35(2): e00009919. https://doi.org/10.1590/0102-311×00009919

22. Foodand Agriculture Organization of the United Nations. FAO, IFAD, UNICEF, WFP and WHO. 2018. The State of Food Security and Nutrition in the World 2018. Building climate resilience for food security and nutrition. Rome: FAO. 2018.

23. Castro IRR. Má nutrição, iniquidade e a garantia do Direito Humano à Alimentação Adequada. Cien Saude Colet. 2019; 24(7): 2376-2376. https:// doi.org/10.1590/1413-81232018247.15392019

24. Dinour LM, Bergen D, Yeh MC. The food insecurityobesity paradox: a review of the literature and the role food stamps may play. J Am Diet Assoc. 2007; 107(11):195261. $\quad$ https://doi.org/10.1016/j.jada.2007.08.006

25. Kac G, Velasquez-Melendez G, Schlussel MM, SegallCorrêa AM, Silva AA, Pérez-Escamilla R. Severe food insecurity is associated with obesity among Brazilian adolescent females. Public Health Nutr. 2012; 15(10):185460. $\quad$ https://doi.org/10.1017/S1368980011003582

26. Laraia BA. Food insecurity and chronic disease. Adv Nutr. 2013;4(2):203-12. https://doi.org/10.3945/an.112.003277

27. Pérez-Escamilla R, Segall-Corrêa AM, Maranha LK, Sampaio MFA, Marín-León L, Panigassi G. An adapted version of the U.S. Department of Agriculture Food Insecurity Module is a valid tool for assessing household food insecurity in Campinas, Brazil. J Nutr. 2004; 134(8):1923-8. $\quad$ https://doi.org/10.1093/jn/134.8.1923

28. Instituto Brasileiro de Geografia e Estatística. Pesquisa Nacional por Amostra de Domicílios. Segurança Alimentar 2013. Rio de Janeiro: IBGE; 2014 [citado 2020 Ago 22] Disponível em: https:// biblioteca.ibge.gov.br/visualizacao/livros/liv91984.pdf

29. Santos TG, Silveira JAC, Longo-Silva G, Ramires EKNM, Menezes RCE. Tendência e fatores associados à insegurança alimentar no Brasil: Pesquisa Nacional por Amostra de Domicílios 2004, 2009 e 2013. Cad Saude Publica. 2018; 34(4): e00066917. http://dx.doi.org/10.1590/0102-311x00066917

30. Organização das Nações Unidas para a Alimentação e a Agricultura. O estado da segurança alimentar e nutricional noBrasil2015. Brasília:FAO-Brasil;2015[citado2020Set 10]. Disponível em: http://www.fao.org/fileadmin/user_upload/ FAO-countries/Brasil/docs/SOFI_Brasil_2015_final.pdf 
31. Food and Agriculture Organization of the United Nations. The State of Food Insecurity in the World 2015. Meeting the 2015 international hunger targets: taking stock of uneven progress. Rome: FAO; 2015 [cited 2020 Sep 10]. Available from: http://www.fao.org/3/a-i4646e.pdf

32. Organização das Nações Unidas. Crescimento da renda dos 20\% mais pobres ajudou Brasil a sair do mapa da fome, diz ONU; 2015 [acesso 2020 Jul 10]. Disponível em: http:// nacoesunidas.org/crescimento-da-renda-dos-20-maispobres-ajudou-brasil-a-sair-do-mapa-da-fome-diz-onu/

33. Vasconcelos FAG, Machado ML, Medeiros MAT, Neves $J A$, RecineE, Pasquim EM. Public policies of foodand nutrition in Brazil: From Lula to Temer. Rev Nutr. 2019; 32: e180161. https://doi.org/10.1590/1678-9865201932e180161

34. Instituto Brasileiro de Geografia e Estatística. Pesquisa de Orçamentos Familiares 2017-2018: Análise da segurança alimentar no Brasil. Rio de Janeiro: IBGE; 2020 [citado 2020 Set 22]. Disponível em: https:// biblioteca.ibge.gov.br/visualizacao/livros/liv101749.pdf

35. Organização Pan-Americana de Saúde. Brasil. Folha informativa - COVID-19 (doença causada pelo novo coronavírus). Brasília: OPAS; 2020 [citado 2020 Set 20]. Disponível em: https:// www.paho.org/pt/covid 19\#: : text=A\% 20 Organiza\% C 3\%A7\% C 3\% A30\%20Mundial\%20 da $\% 20 \mathrm{Sa} \%$ C $3 \%$ BAde,previsto $\% 20$ no $\% 20$ Regulamento\%20Sanit\%C3\%A1rio\%20Internacional.

36. Brasil. Ministério da Saúde. Coronavírus Brasil. COVID-19. Painel Coronavírus. [Internet]. Brasília: Ministério da Saúde; 2020 [citado 2020 Set 30]. Disponível em: https://covid.saude.gov.br/

37. FIAN-Brasil. Organização pelo Direito Humano à Alimentação e à Nutrição Adequadas. Impacto da Covid-19 na Realização do Direito Humano à Alimentação e à Nutrição Adequadas. Relatório preliminar de monitoramento. Brasília: Fian-Brasil; 2020 [citado 2020 Set 10]. Disponível em: https://fianbrasil.org.br/wp-content/uploads/2020/04/ Relatorio-covid-19-fian-internacional-formatado.pdf

38. Boletins da Faculdade de Saúde Pública. Mais frutas, hortaliças e feijão entraram na mesa do brasileiro durante a pandemia, mostra Estudo NutriNet Brasil. Faculdade de Saúde Pública. Universidade de São
Paulo, 2020 [citado 2020 Ago 17]. Disponível em: https://www.fsp.usp.br/site/noticias/mostra/22097

39. Empresa Brasileira de Pesquisa Agropecuária. Consumo de hortaliças na quarentena, 2020 [citado 2020 Set 08]. Disponível em: https://www. embrapa.br/hortalicas/pesquisa-consumo-covid19

40. Fundo das Nações Unidas para a Infância. Impactos primários e secundários da COVID-19 em Crianças e Adolescentes. IBOPE Inteligência, 2020 [citado 2020 Set 10]. Disponível em: https:// www.unicef.org/brazil/media/9966/file/impactoscovid-criancas-adolescentes-ibope-unicef-2020.pdf

41. Segall-Corrêa AM, Marin-Leon L, Helito H, PérezEscamilla R, Santos LMP, Paes-Sousa R. Transferência de renda e segurança alimentar no Brasil: análise dos dados nacionais. Rev Nutr. 2008; 21(Suppl): 39s-51s. Available from: $\quad$ http://www.scielo.br/scielo.php?script=sci arttext\&pid=S1415-52732008000700005\&lng=en.

42. Palmeira PA, Salles-Costa R, Pérez-Escamilla R. Effects of family income and conditional cash transfers on household food insecurity: evidence from a longitudinal study in Northeast Brazil. Public Health Nutr. 2020; 23(4):756767. $\quad$ https://doi.org/10.1017/S1368980019003136

43. Menezes-Filho NA. Pandemia, pobreza e política. 2020 [citado 2020 Jul 10]. Disponível em: https://valor.globo. com/opiniao/coluna/pandemia-pobreza-e-politica.ghtml

44. Brasil. Câmara dos Deputados. Projeto de Lei $N^{\circ}$ 9236/2017: Altera o $\S 11$ e acrescenta os $\S \S 12,13,14$ e 15 ao art. 20 da Lei n 8.742, de 7 de dezembro de 1993, para dispor sobre parâmetros adicionais para caracterização da situação de vulnerabilidade social, para fins de elegibilidade ao Benefício de Prestação Continuada, pela pessoa com deficiência ou idosa. 2020 [citado Set 10]. Disponível em:https://www.camara.leg.br/proposicoesWeb/ $\underline{\text { fichadetramitacao? idProposicao }=2163972}$

45. Burity $V$, Franceschini $T$, Valente $F$, Recine $E$, Leão $M$, Carvalho MF. Direito humano à alimentação adequada no contexto da segurança alimentar e nutricional. Brasília: ABRANDH; 2010 [citado 2020 Set 15]. Disponível em: http://www.mds.gov.br/webarquivos/ publicacao/seguranca alimentar/DHAA SAN.pdf 
46. Müller AL, Silva MK, Schneider S. A construção de políticas públicas para a agricultura familiar no Brasil: o programa de aquisição de alimentos. Estudos Sociedade e Agricultura, v. 20, Rio de Janeiro, 2012. In: A Contribuição brasileira à segurança alimentar e nutricional sustentável [recurso eletrônico]/organizadores Potira V. Preiss, Sergio Schneider e Gabriela Coelhode-Souza. Porto Alegre: Editora da UFRGS, 2020.

47. Instituto de Pesquisa Econômica Aplicada. O Programa de Aquisição de Alimentos (PAA): instrumento de dinamismo econômico, combate à pobreza e promoção da segurança alimentar e nutricional em tempos de covid-19. Diretoria de Estudos e Políticas Regionais, Urbanas e Ambientais. Nota técnica $\mathrm{n}^{\circ}$ 17. Brasília: IPEA; 2020 [citado 2020 Set 15]. Disponível em: https:// www.ipea.gov.br/portal/index.php?option $=\mathrm{com}$ content \&view $=$ article $\& i d=35581 \& 1$ te $\operatorname{mid}=448$

48. FIAN-Brasil. Organização pelo Direito Humano à Alimentação e à Nutrição Adequadas. Informe Dhana 2019: autoritarismo, negação de direitos e fome. Brasília: Fian-Brasil, 2019 [citado 2020 Set 02]. Disponível em: https://fianbrasil.org.br/wp-content/ uploads/2019/11/Informe-Dhana-2019 v-final.pdf

49. Food and Agriculture Organization of the United Nations. Mitigating impacts of Covid-19 on food trade and markets. Rome: FAO; 2020 [cited 2020 Sep 10]. Available from: http://www.fao.org/news/story/pt/item/1268719/icode/

50. Organização das Nações Unidas para a Alimentação e a Agricultura. FAO no Brasil. 25 países de América Latina e do Caribe se coordenam para apoiar o funcionamento regular do sistema alimentar durante a crise da Covid-19. 2020 [citado 2020 Ago 10]. Disponível em: http:// www.fao.org/brasil/noticias/detail-events/pt/c/1269594/

51. Confederação da Agricultura e Pecuária do Brasil. Boletim CNA: impacto do coronavírus. 2020 [citado 2020 Ago 10]. Disponível em: https://www.cnabrasil. org.br/noticias/boletim-cna-impactos-do-coronavirus

52. Brasil. Lei $n^{\circ}$ 11.947, de 16 de junho de 2009. Dispõe sobre o atendimento da alimentação escolar e do Programa Dinheiro Direto na Escola aos alunos da educação básica. Diário Oficial da União, Brasília, 17 jun.

53. Brasil. Ministério da Agricultura Pecuária e
Abastecimento. Ministério da Educação. Agência Nacional de Assistência Técnica e Extensão Rural. Orientações para a execução do PNAE durante a situação de emergência decorrente da pandemia do coronavírus (covid-19). Brasília: MAPA; 2020 [citado 2020 Set 10]. Disponível em: https://www.gov.br/agricultura/pt-br/ campanhas/mapacontracoronavirus/documentos/ cartilha-orientacoes-para-a-execucao-do-pnae/view

54. Brasil. Ministério da Educação. Fundo Nacional para o Desenvolvimento da Educação. Entrega de kits da alimentação escolar continua garantida durante a pandemia. 2020 [citado 2020 Ago 10]. Disponível em: https://www.fnde.gov.br/index.php/acesso-a-informacao/ institucional/area-de-imprensa/noticias/item/13554entrega-de-kits-da-alimenta \% C 3\%A7\% C 3\%A30escolar-continua-garantida-durante-a-pandemia

55. Centro Colaborador de Alimentação e Nutrição Escolar do Instituto Federal do Sul de Minas CECANE. Experiências exitosas do PNAE durante a pandemia/20. 2020 [citado 2020 Ago 10]. Disponível em: $\quad$ https://cecane.ifsuldeminas.edu.br/2020/06/03/ experiencias-exitosas-municipio-de-nacip-raydan/

56. Rede Brasileira de Alimentação e Nutrição do Escolar. Experiências exitosas: PNAE em tempos de covid-19: relato de caso do município de Montenegro/ RS. 2020 [citado 2020 Ago 10]. Disponível em: https://rebrae.com.br/index.php/experiencias/ experiencias-exitosas/367-pnae-em-tempos-de-covid19-relato-de-caso-do-municipio-de-montenegro-rs

57. São Paulo. Cidade de São Paulo. Secretaria Municipal de Educação. Cartão Merenda. 2020 [citado 2020 Ago 10]. Disponível em: https:// educacao.sme.prefeitura.sp.gov.br/cartao-merenda/

58. Global Panel on Agriculture and Food Systems for Nuatrition. Food systems and diets: Facing the challenges of the 21st century. London: WHO; 2016 [cited 2020 Sep 10]. Available from: http://ebrary.ifpri.org/utils/ getfile/collection/p15738coll5/id/5516/filename/5517.pdf

59. Jaime PC, Delmuè DCC, Campello T, Silva DO, Santos LMP. Um olhar sobre a agenda de alimentação e nutrição nos trinta anos do Sistema Único de Saúde. Cien Saude Colet. 2018; 23(6):1829-1836. http:// dx.doi.org/10.1590/1413-81232018236.05392018 
60. High Level Panel of Experts on Food Security and Nutrition. Nutrition and food systems. A report by the High Level Panel of Experts on Food Security and Nutrition of the Committee on World Food Security. Rome: High Level Panel of Experts on Food Security and Nutrition; 2017 [cited 2020 Ago 30]. Available from: http://www.fao.org/3/a-i7846e.pdf

61. Monteiro CA, Cannon G. The role of the transnational ultra-processed food industry in the pandemic of obesity and its associated diseases: problems and solutions. World Nutrition. 2019; 10(1):8999. $\quad$ https://doi.org/10.26596/wn.201910189-99

62. Organização Pan-Americana da Saúde. Folha informativa COVID-19 - Escritório da OPAS e da OMS no Brasil, 2020 [citado 2020 Set 14]. Disponível em: https://www.paho.org/pt/covid19\#: : :text=0\%20 risco $\% 20$ de $\% 20$ exposi $\%$ C 3 \% A7\% C 3\%A30\% 20 ocupacional,com $\% 20$ superf $\%$ C $3 \%$ ADcies $\% 20$ e $\% 20$ objetos $\% 20$ contaminados.

63. Fórum Brasileiro de Soberania e Segurança Alimentar e Nutricional. Garantir o direito à alimentação e combater a fome em tempos de coronavírus: a vida e a dignidade humana em primeiro lugar, 2020 [citado 2020 Ago 10]. Disponível em: https://fbssan.org.br/wp-content/ uploads/2020/04/2020-recomendacoes-sobre-direitoalimentacao-no-contexto-da-covid-versao-2-de-abril-.pdf

64. Rocha E. A Constituição Cidadã e a institucionalização dos espaços de participação social: avanços e desafio. IN: Vaz FT, Musse JS, Santos RF (coords). 20 Anos da Constituição Cidadã: Avaliação e desafio da seguridade social. Brasília: ANFIP, 2008.

65. SousaLRM, Segall-CorrêaAM, VilleAS, Melgar-Quiñonez $\mathrm{H}$. Food security status in times of financial and political crisis in Brazil. Cad Saude Publica. 2019; 35(7):e00084118. http://dx.doi.org/10.1590/0102-311x00084118

66. Sousa LRM, Ville AS, Segall-Corrêa AM, MelgarQuiñonez $H$. Health inequalities and well-being in times of financial and political crisis in Brazil, a case study. Global Public Health. 2019; 14(12):1815-1828. https://doi.org/10.1080/17441692.2019.1616800
67. COVID-19 in Brazil: "So what?". Editorial. Lancet. 2020; 395(10235):1461. https:// doi.org/10.1016/50140-6736(20)31095-3 